\title{
Exercise Intolerance, Lactic Acidosis, and Abnormal Cardiopulmonary Regulation in Exercise Associated with Adult Skeletal Muscle Cytochrome c Oxidase Deficiency
}

\author{
Ronald G. Haller, Steven F. Lewis, Ronald W. Estabrook, Salvatore DiMauro, Serenella Servidei, and Daniel W. Foster \\ Departments of Neurology, Physiology, Biochemistry, and Internal Medicine, The Veterans Administration Medical Center \\ and the University of Texas Southwestern Medical Center, Dallas, Texas 75216; and Department of Neurology, \\ Columbia University College of Physicians and Surgeons, New York, New York 10032
}

\begin{abstract}
A 27-yr-old woman with lifelong severe exercise intolerance manifested by muscle fatigue, lactic acidosis, and prominent symptoms of dyspnea and tachycardia induced by trivial exercise was found to have a skeletal muscle respiratory chain defect characterized by low levels of reducible cytochromes $a$ $+a_{3}$ and $b$ in muscle mitochondria and marked deficiency of cytochrome $c$ oxidase (complex IV) as assessed biochemically and immunologically. Investigation of the pathophysiology of the exercise response in the patient revealed low maximal oxygen uptake ( $1 / 3$ that of normal sedentary women) in cycle exercise and impaired muscle oxygen extraction as indicated by profoundly low maximal systemic arteriovenous oxygen difference $(5.8 \mathrm{ml} / \mathrm{dl}$; controls $=15.4 \pm 1.4$, mean $\pm S D)$. The increases in cardiac output and ventilation during exercise, normally closely coupled to muscle metabolic rate, were markedly exaggerated (more than two- to threefold normal) relative to oxygen uptake and carbon dioxide production accounting for prominent tachycardia and dyspnea at low workloads. Symptoms in our patient are similar to those reported in other human skeletal muscle respiratory chain defects involving complexes I and III, and the exaggerated circulatory response resembles that seen during experimental inhibition of the mitochondrial respiratory chain. These results suggest that impaired oxidative phosphorylation in working muscle disrupts the normal regulation of cardiac output and ventilation relative to muscle metabolic rate in exercise.
\end{abstract}

\section{Introduction}

Severe intolerance of physical exercise associated with elevated blood lactate levels at rest or with minimal exercise is a wellrecognized syndrome of skeletal muscle mitochondrial disease $(1,2)$. In many cases the underlying biochemical abnormality is undefined, but in others a defect involving the respiratory chain in skeletal muscle at the level of complex 1 (NADH-CoQ oxireductase) or complex III (CoQ-cytochrome $c$ oxireductase) has been identified $(1,2)$. In patients with these disorders of skeletal muscle electron transport, trivial exertion leads to muscle fatigue associated with prominent tachycardia and dys-

Address correspondence to Dr. Ronald G. Haller, Neurology Service (127), Veterans Administration Medical Center, 4500 S. Lancaster Road, Dallas, TX 75216.

Received for publication 9 May 1988 and in revised form 7 March 1989.

J. Clin. Invest.

(c) The American Society for Clinical Investigation, Inc.

0021-9738/89/07/0155/07 \$2.00

Volume 84, July 1989, 155-161 pnea in the absence of hemodynamically significant cardiac disease (3-7). The pathophysiology of this symptom complex is poorly understood.

Circulatory and ventilatory responses to exercise normally are closely coupled to skeletal muscle metabolic rate. Regulation is achieved in part by reflexes that originate in working muscle and are activated by metabolites produced in response to muscle energy demands (8). In certain disorders of muscle energy metabolism, circulatory and ventilatory exercise responses are exaggerated apparently due to anomalous accumulations of regulatory metabolites during muscular work (9-13). Comparable physiologic data are lacking in skeletal muscle electron transport defects.

We identified an adult with a respiratory chain defect in skeletal muscle characterized by low levels of reducible cytochromes $a+a_{3}$ and $b$ and marked deficiency of cytochrome c oxidase (COX, ${ }^{1}$ complex IV). As in previously reported respiratory chain defects involving complex I or III (3-7), the dominant clinical feature in our patient was abnormal exertional muscle fatigue in which systemic symptoms of breathlessness and cardiac palpitations contributed to exercise intolerance. We found that this clinical syndrome was related to a distinctive pattern of exercise pathophysiology in which increases in cardiac output and ventilation from rest to exercise were greatly exaggerated relative to metabolic rate as apparent direct consequences of the severe oxidative defect in skeletal muscle.

\section{Methods}

\section{Clinical history}

The patient was a 27-yr-old woman with lifelong exercise intolerance marked by exertional dyspnea and tachycardia in association with premature fatigue of active muscles. Muscle pain was not prominent and she denied exertional muscle cramping or pigmenturia. Early development was noteworthy for the fact that she walked later $(17 \mathrm{mo})$ than her five siblings (10-14 mo). Her parents recalled that she always avoided physical activity and was the first to tire during family outings. As a child, she was unable to run, skate, or skip rope due to easy fatigue. Poor endurance precluded normal school physical education activities and she recalled that when other children were required to run around the gym, she could only walk, becoming fatigued after circling the gym once. Exercise tolerance mildly worsened as she got older, but she was able to attend school and work as a registered nurse by carefully pacing herself and resting frequently. Diet or ethanol were not noted to affect symptoms.

1. Abbreviations used in this paper: a-v, arteriovenous; COX, cytochrome $c$ oxidase; L/P, lactate/pyruvate; MAP, mean arterial pressure; $\mathrm{PCr}$, phosphocreatine; $\mathrm{Pi}$, inorganic phosphate; $\mathrm{RER}$, respiratory exchange ratio; $\mathrm{S}$, systolic. 
The patient came to medical attention during a vacation outing. Toward the end of the day she became separated from a member of her party, resulting in anxiety, a flurry of walking, and inability to rest despite symptoms of shortness of breath and fatigue. She developed severe dyspnea, palpitations, and leg heaviness. After resting she felt better, but symptoms rapidly recurred with a two-block walk to the car. She was so dyspneic she was unable to speak and simply nodded when asked if she wanted to be taken to an emergency room. There she was found to have a severe metabolic acidosis which subsequent testing proved to be lactic acidosis. There was no family history of similar symptoms. Her parents and five siblings were living and well.

On physical examination the patient was small (height, $161 \mathrm{~cm}$; weight, $49.9 \mathrm{~kg}$ ) and thinly muscled. Cardiopulmonary examination was normal. Cranial nerves were intact. There was mild neck flexor and proximal limb weakness with slight difficulty arising from a squat. Recent bilateral bunion surgery contributed to some trouble walking on heels and toes. She was able to perform a sit-up without difficulty. Vibratory perception and proprioception were mildly impaired at the toes and there was slight unsteadiness on tandem walking. Cerebellar function was otherwise normal and reflexes were intact. Normal laboratory tests included hematocrit, hemoglobin, thyroid function tests, serum electrolytes, calcium, magnesium, glucose, urinalysis, and urinary amino acid profile. Serum creatine kinase and aspartate and alanine transaminases were not elevated. Electrocardiogram and chest film were normal, as were electromyogram and motor nerve conduction velocities.

\section{Evaluation}

Cycle exercise. The patient and eight age-matched $(26 \pm 4 \mathrm{yr}$, mean $\pm S D$; wt, $59.8 \pm 7.3 \mathrm{~kg}$ ) healthy women exercised using an electrically braked, pedal-rate independent cycle ergometer (NASA, Skylab). Submaximal and maximal workloads of 5-6 min duration were performed in ascending order of intensity with a 15 -min rest period between each workload. Maximal exercise was regarded as the highest workload at which cycling could be continued for $5 \mathrm{~min}$. At rest and in the last minute of each workload, expired air was collected in Douglas bags and cardiac output ( $\dot{Q})$ was determined. Ventilation ( $\dot{V} E)$, oxygen uptake $\left(\mathrm{V}_{2}\right)$, and carbon dioxide production $\left(\dot{\mathrm{V}} \mathrm{CO}_{2}\right)$ were measured using a Tissot spirometer and mass spectrometer (model 1100A; Perkin-Elmer Corp., Norwalk, CT). The respiratory exchange ratio (RER; $\left.\dot{\mathrm{V}} \mathrm{CO}_{2} / \dot{\mathrm{VO}}_{2}\right), \dot{\mathrm{VE}} / \dot{\mathrm{V}}_{2}$, and $\dot{\mathrm{VE}} / \dot{\mathrm{V}}_{\mathrm{CO}_{2}}$ were calculated from gas exchange data. Cardiac output was measured noninvasively using acetylene rebreathing (14). In this procedure subjects rebreathe a gas mixture consisting of $30 \% \mathrm{O}_{2}, 16 \% \mathrm{He}, 0.7 \% \mathrm{C}_{2} \mathrm{H}_{2}$, and $0.3 \% \mathrm{CO}$ in $\mathrm{N}_{2}$ from an anesthesia bag. Helium serves as an indicator of system volume, and the rate of disappearance of $\mathrm{C}_{2} \mathrm{H}_{2}$ from the system relative to $\mathrm{He}$ is proportional to pulmonary blood flow and $\mathrm{Q}$. Comparison of the acetylene method with simultaneous dye-dilution cardiac output measurements at rest and during exercise established the absence of systematic error, a linear $r$ of 0.94 , and an SE of measurement of $<5 \%$ (14). The increase in cardiac output ( $\Delta \dot{Q}$, liters/minute) from rest to exercise relative to the increase in oxygen uptake (liters/minute; i.e., $\Delta \dot{Q} / \Delta \dot{\mathbf{V}} \mathrm{O}_{2}$ ) was determined as the slope of $\dot{Q}$ on $\dot{\mathrm{V}} \mathrm{O}_{2}$ determined by linear regression analysis of resting, submaximal, and maximal exercise data for each subject. Systemic arteriovenous oxygen difference was calculated as the ratio $\dot{\mathrm{VO}}_{2}$ (milliliters)/Q(deciliters). Heart rate was monitored continuously from electrocardiographic recordings and BP was determined at rest and every $30 \mathrm{~s}$ during exercise using an electrosphygmomanometer (model PE 300; Narco Bio-Systems, Inc., Houston, TX). Venous (antecubital) blood was collected at rest and in the last minute of each workload in the patient and five control subjects. Lactate and pyruvate were determined in perchloric acid extracts of whole blood (15) and the lactate/pyruvate ratio (L/P) was calculated.

Morphological, biochemical, and immunologic evaluation of muscle. An open biopsy of the vastus lateralis muscle was performed for histopathological and biochemical determinations. Portions of the biopsy were frozen in liquid nitrogen-cooled isopentane for histochemistry, fixed in glutaraldehyde for electron microscopy, frozen in liquid nitrogen for subsequent biochemical assays, and used for preparation of fresh skeletal muscle mitochondrial fractions for cytochrome determinations (16). The remainder of the mitochondrial fraction was frozen for assay of selected mitochondrial enzymes. Controls for biochemical and immunologic studies were patients undergoing muscle biopsy who ultimately were deemed to be free of neuromuscular disease and normal subjects undergoing hip replacement.

Cytochrome difference (reduced-oxidized) spectra were determined at room temperature on a dual beam spectrophotometer (model DW-2a; American Instrument Co., Silver Spring, MD) with cytochromes reduced using substrate (pyruvate, succinate) or sodium dithionite (17). Gassing with carbon monoxide revealed no spectral shift, indicating that the mitochondrial preparations were free of contaminating hemoglobin and myoglobin. Cytochrome concentrations were calculated using the extinction coefficients used by Bookleman et al. (17). The activities of NADH-dehydrogenase (NADH ferricyanide reductase, EC 1.6.5.3) (18), NADH-cytochrome $c$ reductase (NADHcytochrome $c$ oxidoreductase, EC 1.6.99.3) \pm rotenone (19), succinatecytochrome $c$ reductase (19), succinate dehydrogenase (EC 1.3.99.1) (20), COX (ferrocytochrome $c$ : oxygen oxidoreductase, EC 1.9.3.1) (21), and citrate synthase (EC 4.1.3.7) (22) were determined spectrophotometrically in freeze-thawed, sonicated mitochondrial extracts. Oligomycin-sensitive mitochondrial ATPase (ATP synthase, EC 3.6.1.34) was assayed by monitoring phosphate liberation at $30^{\circ} \mathrm{C}(23)$. Mitochondrial protein was determined colorometrically (24). In freeze-thawed, sonicated crude muscle homogenates, activities of citrate synthase and COX were determined spectrophotometrically (25). Carnitine palmitoyl transferase (palmitoyl-CoA: L-carnitine-o-palmitoyl transferase, EC 2.3.1.21) activity was assayed using the "forward reaction" (reaction II of McGarry and Foster) (26). Carnitine (free and total) was assayed radiochemically in whole muscle homogenates (27).

Polyclonal antibodies were raised in rabbits against COX purified from human heart (25). The presence of enzymatically inactive, immunologically crossreacting material was estimated by ELISA using muscle homogenates and mitochondrial preparations as antigen and different dilutions of antiserum against COX.

\section{Results}

Physiology. Oxygen uptake was normal at rest and rose normally in relation to exercise workload (Table I and Fig. 1). However, maximal oxygen uptake $(10.7 \mathrm{ml} / \mathrm{kg}$ per min) and workload $(20 \mathrm{~W}, 0.4 \mathrm{~W} / \mathrm{kg})$ were markedly low in comparison to values for healthy women $(31.6 \pm 3.9 \mathrm{ml} / \mathrm{kg}$ per $\mathrm{min}$ and $138 \pm 22 \mathrm{~W}, 2.3 \pm 0.4 \mathrm{~W} / \mathrm{kg}$, respectively, mean $\pm \mathrm{SD}$ ).

Cardiac output and heart rate were normal at rest but during exercise increased dramatically relative to oxygen uptake (Table I and Fig. 2). The slope of increase in cardiac output in relation to oxygen uptake, i.e., $\Delta \dot{\mathrm{Q}} / \Delta \dot{\mathrm{V}}_{2}$, was more than threefold greater than normal (patient, 16.2; controls, $5.1 \pm 0.7$ ), indicating a profound disturbance of the normal relationship between $\dot{Q}$ and $\mathrm{V}_{2}$ in exercise (Fig. 2 B). Maximal cardiac output was only slightly lower than that of control women $(180.3 \mathrm{ml} / \mathrm{kg}$ per min vs. $208 \pm 14.1)$. Systolic (S) and mean arterial pressures (MAP) at rest (patient: $S=117$, MAP $=89 \mathrm{mmHg}$; controls: $\mathrm{S}=123 \pm 9, \mathrm{MAP}=89 \pm 9 \mathrm{mmHg}$ ) and with maximal exercise (patient: $S=152$, MAP $=117$; controls: $S=166 \pm 10$, MAP $=113 \pm 13$ ) were comparable in the patient and control women, but the increase in arterial pressure relative to workload was abnormally steep in the patient. Systemic arteriovenous oxygen $\left(\mathrm{a}-\mathrm{v} \mathrm{O}_{2}\right)$ difference rose approximately threefold from 5.4 to $15.4 \mathrm{ml} / \mathrm{dl}$ from rest to maximal exercise in normal women, but there was only a $25 \%$ increase in oxygen extraction per unit of blood during exercise in the patient (rest $=4.6, \max =5.8$ ) (Table I), implying that impaired oxygen extraction by working muscle was a principal 
Table I. Circulation and Gas Exchange at Rest and in Maximal Exercise

\begin{tabular}{|c|c|c|c|c|c|c|c|c|c|c|c|c|c|}
\hline & Work & $\dot{\mathrm{V} E}$ & $\dot{\mathrm{V}}_{2}$ & $\dot{\mathrm{V} E} / \dot{\mathrm{V}}_{2}$ & $\dot{\mathrm{VE}} / \mathbf{\mathrm { V }} \mathrm{CO}_{2}$ & RER & $\dot{\mathrm{Q}}$ & HR & $\begin{array}{l}\text { Syst a-v } \\
\mathrm{O}_{2} \text { diff }\end{array}$ & $\Delta \dot{\mathrm{Q}} / \Delta \dot{\mathrm{V}}_{2}$ & Lact & Pyr & $\mathrm{L} / \mathrm{P}$ \\
\hline & $w$ & liters/min & $\mathrm{ml} / \mathrm{kg}$ per min & & & & $\mathrm{ml} / \mathrm{kg}$ per min & $b p m$ & $m l / d l$ & & $m M$ & $m M$ & \\
\hline \multicolumn{14}{|l|}{ Rest } \\
\hline Control mean & - & 10.1 & 4.3 & 40.1 & 49.6 & 0.81 & 79.4 & 82 & 5.41 & - & 0.81 & 0.09 & 9.8 \\
\hline SD & & 1.3 & 0.7 & 5.9 & 8.2 & 0.11 & 6.9 & 14 & 0.66 & & 0.27 & 0.03 & 3.1 \\
\hline Patient & - & 12.0 & 4.3 & 55.0 & 63.7 & 0.87 & 94.7 & 89 & 4.63 & - & 2.45 & 0.13 & 18.3 \\
\hline \multicolumn{14}{|l|}{ Max exercise } \\
\hline Control mean & 135.6 & 79.2 & 31.6 & 42.1 & 36.2 & 1.16 & 208.3 & 186 & 15.37 & 5.1 & 8.81 & 0.18 & 49.9 \\
\hline SD & 21.6 & 16.0 & 3.3 & 6.6 & 5.6 & 0.06 & 14.1 & 5 & 1.40 & 0.7 & 2.12 & 0.03 & 13.1 \\
\hline Patient & 21.0 & 60.6 & 10.7 & 121.3 & 71.5 & 1.70 & 180.3 & 168 & 5.76 & 16.2 & 9.43 & 0.14 & 65.1 \\
\hline
\end{tabular}

$\dot{\mathrm{V}}$, ventilation; $\dot{\mathrm{V}}_{2}$, oxygen consumption; $\dot{\mathrm{V}} \mathrm{CO}_{2}$, carbon dioxide production; $\dot{\mathrm{Q}}$, cardiac output; $\mathrm{HR}$, heart rate; syst a-v $\mathrm{O}_{2}$ diff, systemic arteriovenous $\mathrm{O}_{2}$ difference; $\Delta \dot{\mathrm{Q}} / \Delta \dot{\mathrm{V}} \mathrm{O}_{2}$, increase in cardiac output relative to the increase in oxygen uptake from rest to exercise; Lact, venous lactate; Pyr, venous pyruvate.

feature of the patient's low maximal oxygen uptake and excessive oxygen transport.

Ventilation was normal at rest but increased during exercise in great excess relative to $\dot{\mathrm{V}}_{2}$ (maximal $\mathrm{VE} / \dot{\mathrm{V}}_{2}$ : patient $=121.3$, controls $=42.1 \pm 6.6)$ and $\dot{\mathrm{V}} \mathrm{CO}_{2}\left(\operatorname{maximal} \dot{\mathrm{VE}} / \dot{\mathrm{V}} \mathrm{CO}_{2}\right.$ : patient $=71.5$, controls $=36.2 \pm 5.6)($ Fig. $3, A$ and $B)$. RER was normal at rest but rose abnormally steeply relative to workload and oxygen uptake (Fig. $3 C$ ). Maximal RER was abnormally high (1.70 vs. $1.16 \pm 0.06)$. The patient's resting venous lactate varied substantially (range, $0.85-4.8 \mathrm{mM}$ ) and was elevated except after prolonged inactivity. Venous lactate and the $\mathrm{L} / \mathrm{P}$ after $5 \mathrm{~min}$ of maximal exercise was normal (patient lactate $=9.4 \mathrm{mM}, \mathrm{L} / \mathrm{P}=65 ;$ control lactate $=8.8 \pm 2.1$, $\mathrm{L} / \mathrm{P}=50 \pm 13$ ) but the increase in lactate and $\mathrm{L} / \mathrm{P}$ relative to workload and level of oxygen uptake was excessive (Fig. 4).

Histology. Routine muscle histochemistry showed only minor abnormalities; modified Gomori trichrome revealed a slight increase in internal red staining but no striking subsarcolemmal accumulations indicative of "ragged red" fibers (28). Moderately increased lipid accumulation predominantly in type 1 fibers was apparent on oil-red-O staining. The histochemical stain for cytochrome oxidase was absent in virtually all fibers $(>90 \%)$ in the biopsy specimen. Abundant lipid droplets and glycogen granules were apparent ultrastructurally. Mitochondria were abundant but appeared morphologically normal.

Biochemistry. In isolated mitochondria, levels of reducible cytochromes $a+a_{3}$ and $b$ were markedly low while cytochrome $c+c_{1}$ was increased compared with controls (Fig. 5, Table II). COX activity was strikingly reduced to $10 \%$ and succinate-cytochrome $c$ reductase to $30 \%$ of the mean of con-

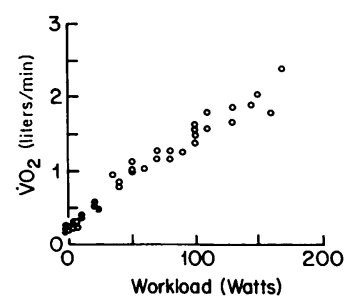

Figure 1. Oxygen uptake $\left(\dot{\mathrm{V}}_{2}\right)$ relative to exercise workload as plotted at rest $(0 \mathrm{~W})$ and during cycle exercise for normal women (open circles) and the patient (solid circles). Normal women $(n=8)$ were studied at rest and during exercise at three workloads with all testing completed during a single testing session. Results for the patient represent three exercise sessions performed on separate days. trols (Table II). In contrast, activities of other respiratory chain enzymes, including NADH dehydrogenase, rotenone-sensitive NADH-cytochrome $c$ reductase, succinic dehydrogenase, and oligomycin-sensitive ATPase, were normal or increased compared with control values (Table II). Citrate synthase activity in isolated mitochondria was three times the control mean. In crude muscle homogenates $\mathrm{COX}$ activity was low, while levels of carnitine palmitoyl transferase and citrate synthase were two- to threefold the mean for control subjects (Table II). Levels of free (patient $=0.63 \mu \mathrm{mol} / \mathrm{g}$ wet wt, control $=1.80 \pm 0.39 \mu \mathrm{mol} / \mathrm{g}$ wet $\mathrm{wt}$ ) and total (patient $=0.91$, control $=2.82 \pm 0.41)$ muscle carnitine were reduced (Table II).

Corresponding to the marked reduction in enzymatically and histochemically measured COX activity, immunoreactive COX material was markedly low by ELISA in crude muscle homogenates and in isolated mitochondria (Fig. 6), indicating reduced levels of immunologically crossreacting enzyme protein.

\section{Discussion}

This patient had a disorder involving respiratory chain complexes III and IV in skeletal muscle. A complex IV defect is manifest by deficiency of reducible cytochrome $a+a_{3}$, low

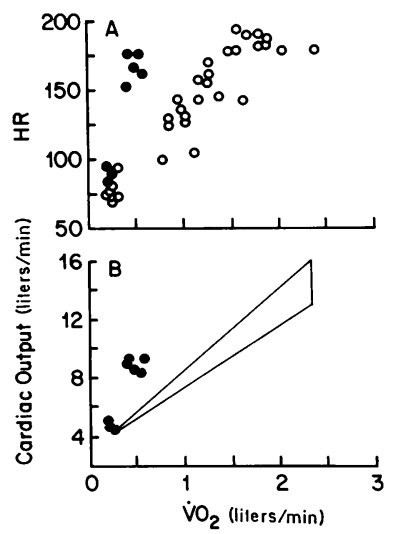

Figure 2. (A) Heart rate (beats per minute) relative to oxygen uptake at rest and during cycle exercise in normal women and the patient. (B) cardiac output relative to oxygen uptake at rest and during exercise. The triangle represents the slope of the increase in cardiac output $(\Delta \dot{\mathrm{Q}})$ relative to the increase in oxygen uptake $\left(\Delta \dot{\mathrm{V}}_{2}\right)$ from rest to exercise for normal women expressed as mean $\pm 2 \mathrm{SD}$. Individual values for the patient obtained in three separate exercise sessions are shown. The mean slopes, $\Delta \dot{\mathrm{Q}} / \Delta \dot{\mathrm{V}}_{2}$, for normal subjects $=5.1 \pm 0.07$; patient $=16.2$. Symbols and exercise conditions are as indicated in Fig. 1. 


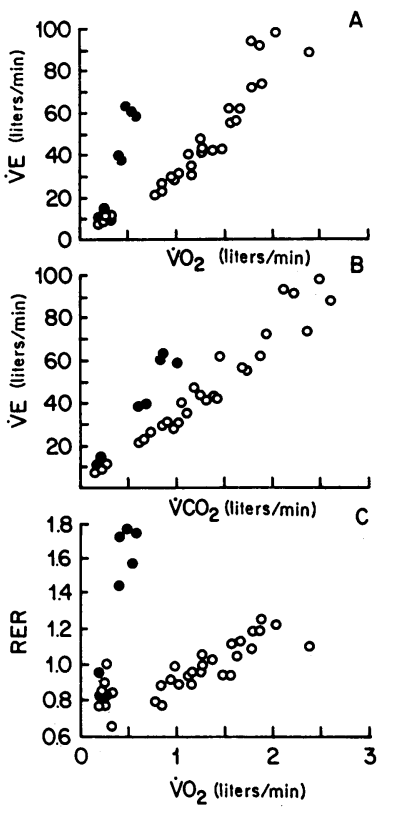

Figure 3. $(A)$ Ventilation (V்) relative to oxygen uptake $\left(\dot{\mathrm{VO}}_{2}\right)$ at rest and during cycle exercise for normal women and the patient. $(B)$ Ventilation relative to carbon dioxide production $\left(\dot{\mathrm{V}} \mathrm{CO}_{2}\right) .(C)$ RER at rest and during cycle exercise in normal women compared with the patient. Symbols and exercise conditions are as indicated in Fig. 1.

COX enzymatic activity, and reduced immunoreactive COX protein. A complex III defect is indicated by deficient reducible cytochrome $b$ and is consistent with reduced activity of succinate-cytochrome $c$ reductase. Activities of other mitochondrial inner membrane or matrix enzymes are normal or increased (carnitine palmitoyl transferase, citrate synthase) compared with control subjects. Increased mitochondrial protein and activities of other oxidative enzymes have been found in muscle respiratory chain defects (29), implying that these disorders promote mitochondrial biogenesis. This may compensate partially for the oxidative defect as illustrated by the fact that in our patient COX activity in whole muscle was higher ( $20 \%$ of normal) than that expressed per milligram of mitochondrial protein ( $10 \%$ of normal).

In contrast to most respiratory chain disorders $(1,2)$, typical ragged red fibers, related to subsarcolemmal accumulation of numerous, abnormal mitochondria, were not seen in the muscle biopsy sample, and mitochondrial morphology appeared largely normal. Similar histologic findings have been reported in COX deficiency associated with Leigh syndrome (30), in a patient with fatal infantile COX deficiency (31), and in a patient clinically similar to ours with exercise intolerance and lactic acidosis in whom a mitochondrial disorder was suspected though muscle biochemical investigations were not undertaken (32). Such observations stress the limitations of mus-

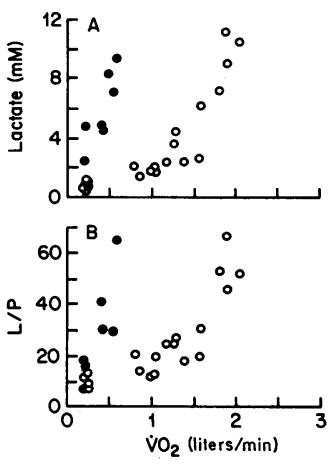

Figure 4. $(A)$ Venous lactate relative to oxygen uptake at rest and during cycle exercise for normal women and the patient. $(B)$ The $\mathrm{L} / \mathrm{P}$ relative to oxygen uptake at rest and during cycle exercise. Symbols and exercise conditions are as indicated in Fig. 1.

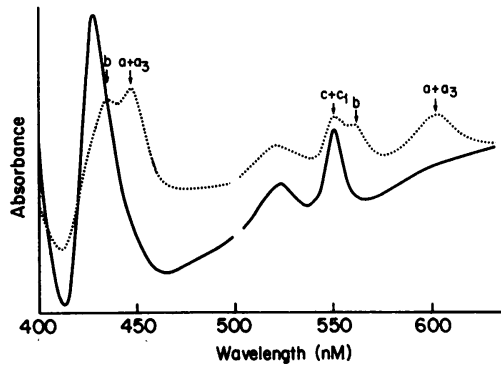

Figure 5. Room temperature cytochrome difference spectra (reduced-oxidized) of muscle mitochondrial preparations from a control subject (dashed line) and the patient (solid line). Absorbance maxima of cytochromes $a$ $+a_{3}, b$, and $c+c_{1}$ are indicated with arrows. cle morphology and the need for more specific histochemical or biochemical determinations to identify muscle mitochondrial defects.

The dominant clinical syndrome of severe exercise intolerance in our patient differs from previously reported cases of COX deficiency (33). Human COX deficiency has been identified most commonly as a fatal infantile myopathy (33) or as a fatal childhood encephalomyopathy in which central nervous system and muscle symptoms coexist $(30,33,34)$. Combined deficiency of complexes IV and III, as found in our patient, is common in fatal infantile COX deficiency (33). Pleiotropic respiratory chain defects involving cytochromes $a+a_{3}$ and $b$ may occur in yeast with either mitochondrial or nuclear gene defects (35). Mitochondrial DNA codes for at least 13 proteins including 3 subunits of cytochrome oxidase and cytochrome $b$ as well as subunits in complex I and mitochondrial (oligomycin-sensitive) ATPase. The remaining respiratory chain proteins, including those that may confer tissue specificity (36), are coded on the nuclear genome. About half of reported cases of COX deficiency are familial with apparent autosomal recessive inheritance, implying a nuclear genomic defect. None

Table II. Mitochondrial Enzymes/Cytochromes

\begin{tabular}{|c|c|c|c|}
\hline & Patient & Control \pm SD & $n$ \\
\hline \multicolumn{4}{|l|}{$\begin{array}{l}\text { Isolated mitochondria }(\mu \mathrm{mol} / \mathrm{min} \\
\quad \text { per } \mathrm{mg} \text { protein) }\end{array}$} \\
\hline NADH dehydrogenase & 2,600 & $1,930 \pm 590$ & (38) \\
\hline $\begin{array}{l}\text { NADH-cytochrome } c \text { reductase, } \\
\text { rotenone sensitive }\end{array}$ & 110 & $120 \pm 39$ & (28) \\
\hline Succinate-cytochrome $c$ reductase & 72 & $244 \pm 65$ & (34) \\
\hline Cytochrome $c$ oxidase & 140 & $1,340 \pm 320$ & (23) \\
\hline ATPase (oligomycin sensitive) & 24.8 & $16: 9 \pm 7.8$ & (6) \\
\hline Citrate synthase & 1,103 & $375 \pm 110$ & (21) \\
\hline Succinate dehydrogenase & 235 & $153 \pm 51$ & (21) \\
\hline \multicolumn{4}{|l|}{ Cytochromes (nmol/mg protein) } \\
\hline$b$ & 0.03 & $0.24 \pm 0.06$ & (8) \\
\hline$c+c_{l}$ & 0.57 & $0.38 \pm 0.08$ & (8) \\
\hline$a+a_{3}$ & 0.01 & $0.30 \pm 0.06$ & (8) \\
\hline \multicolumn{4}{|l|}{$\begin{array}{l}\text { Muscle homogenates ( } \mu \mathrm{mol} / \mathrm{min} \text { per } \\
\quad \mathrm{g} \text { tissue) }\end{array}$} \\
\hline Citrate synthase & 29.20 & $10.48 \pm 4.00$ & (19) \\
\hline Carnitine palmitoyl transferase & 24.10 & $12.20 \pm 3.50$ & (12) \\
\hline $\operatorname{cox}$ & 0.58 & $2.85 \pm 0.53$ & (38) \\
\hline Carnitine, total ( $\mu \mathrm{mol} / \mathrm{g}$ tissue) & 0.91 & $2.71 \pm 0.70$ & (18) \\
\hline Carnitine, free ( $\mu \mathrm{mol} / \mathrm{g}$ tissue) & 0.63 & $1.92 \pm 0.61$ & (18) \\
\hline
\end{tabular}




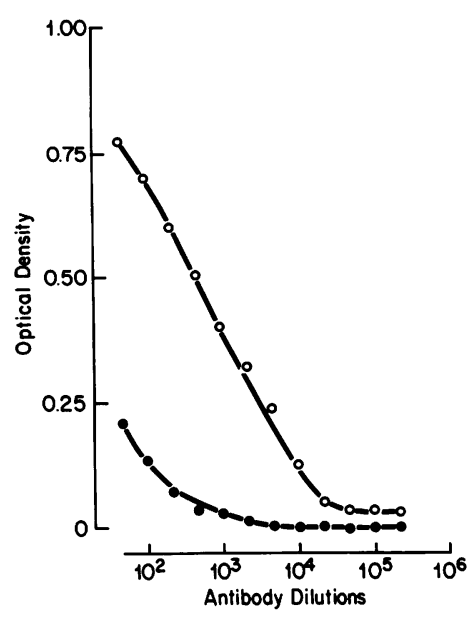

Figure 6. Immunoreactivity by enzyme-linked immunosorbent assay of muscle mitochondrial extracts from the patient (solid circles) and a control (open circles) with progressive dilutions of purified antihuman COX IgG antibody.

had evident maternal inheritance (33) as expected in mitochondrial genome defects, but mitochondrial DNA deletions have been found in the absence of a positive family history in some patients with complex I or III defects (37).

The oxidative defect in our patient severely impairs the capacity for dynamic exercise, underscoring the dominant role of muscle oxidative metabolism in supplying the energy required to sustain exercise. Dependence on anaerobic metabolism to supply muscle energy needs in exercise is correspondingly increased. Anaerobic glycogenolysis and muscle cytoplasmic redox, indicated by levels of blood lactate and L/P, respectively, are high at low levels of muscle work. Similar respiratory chain defects accelerate muscle phosphocreatine depletion during and slow repletion after exercise $(38,39)$.

The nearly $1: 1$ ratio between oxygen transport and utilization in exercise illustrates the normal tight gearing of oxygen delivery to muscle oxidative rate. Assuming normal oxygencarrying capacity (i.e., $\sim 20 \mathrm{ml} \mathrm{O} /$ dl of oxygenated blood), 5 liters of cardiac output are required to transport 1 liter of oxygen in exercise. In normal individuals cardiac output increases 5-6 liters for each liter of increase in oxygen uptake from rest to exercise (i.e., $\Delta \dot{\mathrm{Q}} / \Delta \dot{\mathrm{V}}_{2}=5-6$ ) with only minor variation with respect to age, sex, body weight, level of conditioning, or active muscle mass (40). Mechanisms responsible for matching $\mathrm{O}_{2}$ transport (i.e., $\mathrm{Q}$ ) and $\mathrm{O}_{2}$ utilization include activation of brainstem cardiovascular centers $(a)$ in parallel with activation of motor units via the corticospinal tract, i.e., "central command" (41); and (b) reflexly via unmyelinated afferent fibers that arise in skeletal muscle and are sensitive to metabolites produced during muscle contraction (8).

The muscle oxidative defect in our patient is associated with an increase in cardiac output in exercise that is three times normal relative to the rate of oxidative metabolism, while oxygen extraction per unit of blood (i.e., a-v $\mathrm{O}_{2}$ difference) remains virtually unchanged in contrast to the normal threefold increase from rest to maximal exercise. This indicates a gross mismatch between oxygen transport and utilization in exercise. This is not attributable to anemia or hypermetabolism (42) since hemoglobin concentration and resting oxygen uptake and circulation were normal.

This cardiac response to exercise is inconsistent with primary cardiac disease or cardiac deconditioning. Severe myocardial or valvular disease typically produces a hypokinetic circulation in exercise with low maximal cardiac stroke volume and cardiac output, with normal or high maximal sys- temic a-v $\mathrm{O}_{2}$ difference (43). In our patient, cardiac pump function as indicated by cardiac output, cardiac stroke volume, and arterial pressure in maximal exercise was comparable to normal women. Myocardial work and myocardial metabolic rate are proportional to the product of systolic arterial pressure and heart rate (44). The patient's normal left ventricular performance at a maximal myocardial workload (heart rate $X$ systolic pressure) that is similar to healthy women thus implies that myocardial work and metabolic capacities are broadly normal in striking contrast to the profoundly reduced capacity for skeletal muscle work.

Deconditioning due to physical inactivity lowers maximal cardiac output and stroke volume and increases heart rate at a given workload, but does not change the basic relationship between cardiac output and oxygen uptake in exercise (45). Thus, $\Delta \dot{\mathbf{Q}} / \Delta \dot{\mathrm{V}}_{2}$ remains $5-6$. That the patient's high heart rate in exercise is not simply attributable to deconditioning is implicit in the fact that the cardiac output is threefold normal $\left(\Delta \dot{\mathrm{Q}} / \Delta \dot{\mathrm{VO}}_{2}=16.2\right)$ at a given workload, while cardiac stroke volume is comparable to normal women.

Exaggerated oxygen transport in exercise is not a nonspecific response to muscle pain or weakness as indicated by the finding of a normal $\Delta \dot{\mathrm{Q}} / \Delta \dot{\mathrm{V}}_{2}$ in patients with exertional myalgia, muscular dystrophy, and metabolic myopathies with normal muscle oxidative capacity (11). A normal Q relative to metabolic rate in patients with muscular dystrophy suggests that increased motor unit recruitment at a given level of exercise due to muscle weakness or atrophy does not exaggerate oxygen transport. This implies that increased central command is not the primary mechanism of the exaggerated cardiac output in our patient.

Exercise cardiac output has not been reported heretofore in patients with defined defects in skeletal muscle electron transport, but excessive oxygen transport relative to metabolic rate and impaired extraction of oxygen from blood in exercise has been recognized in other severe muscle oxidative defects. Larsson et al. (9) and Linderholm et al. (10) described patients with an incompletely characterized defect in pyruvate oxidation (high lactate and pyruvate production, low L/P in exercise), and a markedly low maximal oxygen uptake $(\sim 10$ $\mathrm{ml} / \mathrm{kg}$ per $\mathrm{min}$ ). These patients demonstrated excessive increases in cardiac output and muscle blood flow in exercise and markedly subnormal systemic and femoral a-v $\mathrm{O}_{2}$ difference consistent with profoundly impaired muscle oxygen extraction. A steeper than normal rise in cardiac output and blood flow to active muscle relative to oxygen uptake also has been demonstrated in muscle phosphorylase deficiency (McArdle's disease) $(12,46)$ in which the block in glycogen breakdown limits the availability of pyruvate, the substrate required for normal maximal oxidative capacity (47). Thus, McArdle patients have a markedly reduced $\mathrm{V}_{2 \max }$ and a low maximal systemic a-v $\mathrm{O}_{2}$ difference in exercise (48). A direct link between the muscle oxidative defect and exaggerated $\mathrm{O}_{2}$ transport in McArdle's disease is indicated by the fact that increasing muscle oxidative metabolism by augmenting blood levels of glucose or FFA normalizes the excessive heart rate, cardiac output, and muscle blood flow, and increases a-v $\mathrm{O}_{2}$ difference in exercise (49). Conversely, reduced blood levels of oxidative substrate lower $\dot{\mathrm{V}}_{2 \max }$ and oxygen extraction and increase $\Delta \dot{\mathrm{Q}} / \Delta \dot{\mathrm{V}}_{2}$ in McArdle's disease (49).

Experimental respiratory chain inhibition (e.g., amobarbital or cyanide) impairs muscle oxidative phosphorylation and 
oxygen extraction and exaggerates blood flow and cardiac output relative to metabolic rate, reproducing the major metabolic and circulatory abnormalities of our patient $(50,51)$. These results support the hypothesis that severely impaired muscle oxidative phosphorylation is a metabolic common denominator of the exaggerated oxygen transport in exercise in muscle oxidative defects. Nuutinen and co-workers proposed that the muscle phosphorylation potential, [ATP]/[ADP][Pi], an index of oxidative phosphorylation, plays a crucial role in regulating myocardial blood flow (51). They identified a close inverse correlation between [ATP]/[ADP][Pi] and blood flow when oxygen utilization and delivery are closely matched (increased cardiac work or hypoxia) and when oxygen delivery and uptake are dissociated due to inhibition of electron transport (51). Assuming equilibrium of the creatine kinase reaction, the ratio phosphocreatine (PCr)/inorganic phosphate (Pi) is directly proportional to the phosphorylation potential (52). An exaggerated fall in muscle $\mathrm{PCr} / \mathrm{Pi}$ relative to exercise intensity is typical of human respiratory chain defects $(38,39)$, consistent with the hypothesis that the phosphorylation potential in skeletal muscle plays an analogous role in regulating cardiac output in exercise. A greater than normal fall in muscle $\mathrm{PCr} / \mathrm{Pi}$ relative to workload also is a feature of exaggerated $\mathrm{O}_{2}$ transport during exercise in muscle phosphorylase deficiency (53). Notably, increasing oxidative substrate availability in McArdle's disease attenuates the fall in the muscle $\mathrm{PCr} / \mathrm{Pi}$ in parallel with normalization of exercise cardiac output and leg blood flow $(49,53)$. The specific metabolic factors that may link the muscle phosphorylation potential and the circulatory response to exercise are speculative, but likely candidates include increased release of adenosine, $\mathrm{Pi}$, and/or potassium from active muscle $(49,54)$.

Ventilation, like cardiac output, normally is closely coupled to muscle metabolic rate in exercise. Regulation probably involves central command (55) as well as the action of bloodborne muscle metabolites such as $\mathrm{CO}_{2}$ or $\mathrm{H}^{+}$ions. There also may be a reflex ventilatory drive originating in metabolically sensitive neural afferents in working muscle (56). In patients with muscle oxidative defects and exaggerated oxygen transport in exercise, a parallel disturbance of ventilation is typical, marked by normal ventilation at rest but a larger than normal hyperpnea relative to the increase in metabolic rate in exercise. A direct link between muscle oxidative capacity and exercise hyperpnea is suggested by the observation in McArdle's disease that increased oxidative substrate levels normalize exercise ventilation relative to metabolic rate while lowered substrate availability potentiates the exaggerated ventilatory response (13). Compared with McArdle's disease, where lactate production is blocked, hyperpnea is more pronounced and symptoms of exertional dyspnea are more prominent in muscle oxidative defects with exaggerated lactate production in exercise. This is compatible with potentiation of exercise hyperpnea by lactic acidosis. The finding of an abnormally high RER with maximal exercise in our patient is indicative of anomalously high ventilation relative to muscle metabolic rate. Similarly high RER responses to exercise have been noted in patients with complex I deficiency $(3,5)$.

Understanding of the regulatory mechanisms is incomplete, but a coherent hypothesis to explain exaggerated exercise cardiac output and pulmonary ventilation in human disorders of muscle oxidative metabolism is emerging. As illustrated by our patient with an intrinsic muscle respiratory chain defect, a markedly diminished ability to increase muscle oxi- dative phosphorylation relative to energy demand in exercise impairs oxygen extraction and probably promotes a steeper than normal fall in the muscle phosphorylation potential relative to work performed. Exaggerated accumulation of metabolites that normally link oxygen demand, delivery, and utilization via oxidative phosphorylation may result. A parallel system of ventilatory regulation driven by metabolic demand as signalled by the muscle phosphorylation potential and $\mathrm{H}^{+}$production may account for exaggerated ventilatory responses to exercise in muscle oxidative defects.

\section{Acknowledgments}

We thank Marguerite Gunder and Willie Moore for expert technical assistance, Gladys Carter for typing the manuscript, and Dr. C. G. Blomqvist for invaluable support and encouragement.

This work was supported by the Veterans Administration, the Muscular Dystrophy Association, and by National Institutes of Health grants HL-06296, GM-16488, and MO1-RR00633. Dr. Lewis is the recipient of Research Career Development Award HL-01581.

\section{References}

1. Petty, R. K. H., A. E. Harding, and J. A. Morgan-Hughes. 1986. The clinical features of mitochondrial myopathy. Brain. 109:915-938.

2. DiMauro, S., E. Bonilla, M. Zeviani, M. Nakagawa, and D. C. DeVivo. 1985. Mitochondrial myopathies. Ann. Neurol. 17:521-538.

3. Morgan-Hughes, J. A., P. Darveniza, S. N. Kahn, D. N. Landon, R. M. Sherratt, J. M. Land, and J. B. Clark. 1977. A mitochondrial myopathy characterized by a deficiency in reducible cytochrome $b$. Brain. 100:617-640.

4. Land, J. M., J. A. Morgan-Hughes, and J. B. Clark. 1981. Mitochondrial Myopathy-biochemical studies revealing a deficiency of NADH-cytochrome b reductase activity. J. Neurol. Sci. 50:1-13.

5. Edwards, R. H. T., C. M. Wiles, K. Gohil, S. Krywawych, and D. A. Jones. 1982. Energy metabolism in human myopathy. In Disorders of the Motor Unit. D. L. Schotland, editor. John Wiley \& Sons, New York. 715-735.

6. Hayes, D. J., B. R. F. Lecky, D. N. Landon, J. A. MorganHughes, and J. B. Clark. 1984. A new mitochondrial myopathy-biochemical studies revealing a deficiency in the cytochrome b-cl complex (complex III) of the respiratory chain. Brain. 107:1165-1177.

7. Kennaway, N. G., N. R. M. Buist, V. M. Darly-Usmar, A. Papadimitriou, S. DiMauro, R. Kelley, R. A. Capaldi, N. K. Blank, and A. D'Agostino. 1984. Lactic acidosis and mitochondrial myopathy associated with deficiency of several components of complex III of the respiratory chain. Pediatr. Res. 18:991-999.

8. Mitchell, J. H., M. P. Kaufman, and G. A. Iwamoto. 1983. The exercise pressor reflex: its cardiovascular effects, afferent mechanisms and central pathways. Annu. Rev. Physiol. 45:229-242.

9. Larsson, L.-E., H. Linderholm, R. Muller, T. Ringqvist, and R. Sornas. 1964. Hereditary metabolic myopathy with paroxysmal myoglobinuria due to abnormal glycolysis. J. Neurol. Neurosurg. Psychiatry. 27:361-380.

10. Linderholm, H., R. Muller, T. Ringqvist, and R. Sornas. 1969. Hereditary abnormal muscle metabolism with hyperkinetic circulation during exercise. Acta Med. Scand. 185:153-166.

11. Haller, R. G., S. F. Lewis, J. D. Cook, and C. G. Blomqvist. 1983. Hyperkinetic circulation during exercise in neuromuscular disease. Neurology. 33:1283-1287.

12. Lewis, S. F., R. G. Haller, J. D. Cook, and C. G. Blomqvist. 1984. Metabolic control of cardiac output response to exercise in McArdle's Disease. J. Appl. Physiol. 57:1749-1753.

13. Haller, R. G., and S. F. Lewis. 1986. Abnormal ventilation during exercise in McArdle's syndrome: modulation by substrate availability. Neurology. 36:716-719.

14. Triebwasser, J. H., R. L. Johnson, Jr., R. P. Burpo, J. C. Campbell, W. C. Reardon, and C. G. Blomqvist. 1977. Non-invasive 
determination of cardiac output by a modified acetylene rebreathing procedure utilizing mass spectrometer. Aviat. Space Environ. Med. 48:203-209.

15. Lowry, O. H., and J. Passonneau. 1972. A Flexible System of Enzymatic Analysis. Academic Press, New York. 291 pp.

16. Makinen, M. W., and C.-P. Lee. 1968. Biochemical studies of skeletal muscle mitochondria. Arch. Biochem. Biophys. 126:75-82.

17. Bookleman, H., J. M. F. Trijbels, R. C. A. Sengers, and A. J. M. Janssen. 1978. Measurement of cytochromes in human skeletal muscle mitochondria isolated from fresh and frozen stored muscle specimens. Biochem. Med. 19:366-373.

18. King, T. E., and R. L. Howard. 1967. Preparation and properties of soluble NADH dehydrogenase from cardiac muscle. Methods Enzymol. 10:275-294.

19. Sottocasa, G. L., B. Kuylenstierna, L. Ernster, and A. Bergstrand. 1967. An electron transport system associated with the outer membrane of the mitochondria. J. Cell Biol. 32:415-438.

20. King, T. E. 1967. Preparation of succinate dehydrogenase and reconstitution of succinate oxidase. Methods Enzymol. 10:322-331.

21. Wharton, D. C., and A. Tzagoloff. 1967. Cytochrome oxidase from beef heart mitochondria. Methods Enzymol. 10:245-250.

22. Srere, P. A. 1969. Citrate synthase. Methods Enzymol. 13:3-11.

23. Mukherjee, A., and V. S. R. Krishnamurty. 1980. Effect of B-diethylaminoethyl-diphenhyprophylacetate $\mathrm{HCl}$ (SKF-525-A) on canine heart mitochondrial function. Biochem. Pharmacol. 29:283288.

24. Lowry, O. H., N. J. Rosebrough, A. L. Farr, and R. J. Randall. 1951. Protein measurement with the Folin phenol reagent. J. Biol. Chem. 193:265-275.

25. Bresolin, N., M. Zeviani, E. Bonilla, R. H. Miller, R. W. Leech, S. Shanske, M. Nakagawa, and S. DiMauro. 1985. Fatal infantile cytochrome c oxidase deficiency: decrease in immunologically detectable enzyme in muscle. Neurology. 35:802-812.

26. McGarry, J. D., G. F. Leatherman, and D. W. Foster. 1978. Carnitine Palmitoyltransferase I, the site of inhibition of hepatic fatty acid oxidation by malonyl CoA. J. Biol. Chem. 253:4128-4136.

27. McGarry, J. D., and D. W. Foster. 1985. Free and esterified carnitine: the radiometric method. In Methods of Enzymatic Analysis, vol. 8. H. U. Bergmeyer, J. Bergmeyer, and M. Grabl, editors. VCH Publishers, Inc., New York. 474-481.

28. Olson, W., W. K. Engel, G. O. Walsh, and R. Einaugler. 1972. Oculocraniosomatic neuromuscular disease with "ragged-red" fibers. Arch. Neurol. 26:193-211.

29. Hoppel, C. L., D. S. Kerr, B. Dahms, and U. Roessmann. 1987. Deficiency of the reduced nicotinamide adenine dinucleotide dehydrogenase component of complex I of mitochondrial electron transport. $J$. Clin. Invest. 80:71-77.

30. DiMauro, S., S. Servidei, M. Zeviani, M. DiRocco, D. C. DeVivo, S. DiDonato, B. Uziel, K. Berry, B. Hoganson, S. D. Johnsen, and P. C. Johnson. 1987. Cytochrome c oxidase deficiency in Leigh syndrome. Ann. Neurol. 22:498-506.

31. Rimoldi, M., E. Bottachi, L. Rossi, F. Cornelia, G. Uziel, and S. DiDonato. 1982. Cytochrome-c-oxidase deficiency in muscles of a floppy infant without mitochondrial myopathy. J. Neurol. 227:201207.

32. Sussmon, K. E., A. Alfrey, W. M. Kirsch, P. Zweig, P. Felig, and F. Messner. 1970. Chronic lactic acidosis in an adult, a new syndrome associated with altered redox state of certain NAD/NADH coupled reactions. Am. J. Med. 48:104-112.

33. DiMauro, S., M. Zeviani, S. Servidei, E. Bonilla, A. Miranda, A. Prelle, and E. A. Schon. 1986. Cytochrome oxidase deficiency: clinical and biochemical heterogeneity. Ann. NY Acad. Sci. 488:19-32.

34. Arts, W. F. M., H. R. Scholte, M. C. B. Loonen, H. Przyrembel, J. Fernandes, J. M. F. Trijbels, and I. E. M. Luyt-Houwen. 1987. Cytochrome $c$ oxidase deficiency in subacute necrotizing encephalomyopathy. J. Neurol. Sci. 77:103-115.

35. Tzagoloff, A. 1982. Mitochondria. Plenum Publishing Corp., New York. 342 pp.
36. Kuhn-Nentwig, L., and B. Kadenbach. 1985. Isolation and properties of cytochrome $c$ oxidase from rat liver and quantification of immunological differences between isozymes from various rat tissues with subunit-specific antisera. Eur. J. Biochem. 149:147-158.

37. Holt, I. J., A. E. Harding, and J. Morgan-Hughes. 1988. Deletions of mitochondrial DNA in patients with mitochondrial myopathies. Nature (Lond.). 331:717-719.

38. Radda, G. K., P. J. Bore, D. G. Gadian, B. D. Ross, P. Styles, D. J. Taylor, and J. Morgan-Hughes. 1982. 31P NMR examination of two patients with NADH-CoQ reductase deficiency. Nature (Lond.). 295:608-609.

39. Eleff, S., N. G. Kennaway, N. R. M. Buist, V. M. DarleyUsmar, R. A. Capaldi, W. J. Bank, and B. Chance. ${ }^{31}$ P NMR study of improvement in oxidative phosphorylation by vitamins $\mathrm{K}_{3}$ and $\mathrm{C}$ in a patient with a defect in electron transport at complex III in skeletal muscle. Proc. Natl. Acad. Aci. USA. 81:3529-3533.

40. Lewis, S. F., W. F. Taylor, T. M. Graham, J. E. Pettinger, J. E. Schutte, and C. G. Blomqvist. 1983. Cardiovascular responses to exercise as functions of absolute and relative workload. J. Appl. Physiol. 54:1314-1323.

41. Shepherd, J. T., C. G. Blomqvist, A. R. Lind, J. H. Mitchell, and B. Saltin. 1981. Static (isometric) exercise: retrospection and introspection. Circ. Res. 48(Supp 1):179-188.

42. Wade, O. L., and J. M. Bishop. 1962. Cardiac output and regional blood flow. Blackwell Scientific Publications Ltd., Oxford, UK. 185-208.

43. Wilson, J. R., J. L. Martin, D. Schwartz, and N. Ferraro. 1984. Exercise intolerance in patients with chronic heart failure: role of impaired nutritive flow to skeletal muscle. Circulation. 69:1079-1087.

44. Braunwald, E., J. Ross, Jr., and E. H. Sonnenblick. 1976. Mechanisms of contraction of the normal and failing heart. Little, Brown and Company, Boston. 417 pp.

45. Saltin, B., G. Blomqvist, J. H. Mitchell, R. L. Johnson, Jr., K. Wildenthal, and C. B. Chapman. 1968. Response to exercise after bed rest and after training. Circulation. 38(Suppl 7):1-78.

46. Lewis, S. F., R. G. Haller, K. G. Henriksson, N. H. Areskog, and L. Jorfeldt. 1986. Availability of oxidative substrate and leg blood flow during exercise in McArdle's disease. Fed. Proc. 45:783. (Abstr.)

47. Gollnick, P. D. 1985. Metabolism of substrates: energy substrate metabolism during exercise and as modified by training. Fed. Proc. 44:353-357.

48. Haller, R. G., S. F. Lewis, J. D. Cook, and C. G. Blomqvist. 1985. Myophosphorylase deficiency impairs muscle oxidative metabolism. Ann. Neurol. 33:1283-1287.

49. Lewis, S. F., and R. G. Haller. 1986. The pathophysiology of McArdle's disease: clues to regulation in exercise and fatigue. J. Appl. Physiol. 61:391-402.

50. Liang, C.-S., and W. E. Huckabee. 1973. Mechanisms regulating the cardiac output response to cyanide infusion, a model of hypoxia. J. Clin. Invest. 52:3115-3128.

51. Nuutinen, E. M., K. Nishiki, M. Erecinska, and D. F. Wilson. 1982. Role of mitochondrial oxidative phosphorylation in regulation of coronary blood flow. Am. J. Physiol. 243:H159-H169.

52. Chance, B. 1984. Applications of 31P NMR to clinical chemistry. Ann. NY Acad. Sci. 428:318-332.

53. Lewis, S. F., R. G. Haller, J. D. Cook, and R. L. Nunnally. 1985. Muscle fatigue in McArdle's disease studied by 31P NMR: effect of glucose infusion. J. Appl. Physiol. 59:1991-1994.

54. He, M.-X., R. D. Wangler, P. F. Dillon, G. D. Romig, and H. V. Sparks. 1987. Phosphorylation potential and adenosine release during norepinephrine infusion in guinea pig heart. Am. J. Physiol. 253:H1184-H1191.

55. Eldridge, F. L., D. E. Millhorn, and T. G. Waldrop. 1981. Exercise hyperpnea and locomotion: parallel activation from the hypothalamus. Science (Wash. DC). 211:844-846.

56. Liang, C.-S., and W. B. Hood, Jr. 1976. Afferent neural pathway in the regulation of cardiopulmonary responses to tissue hypermetabolism. Circ. Res. 38:209-214. 